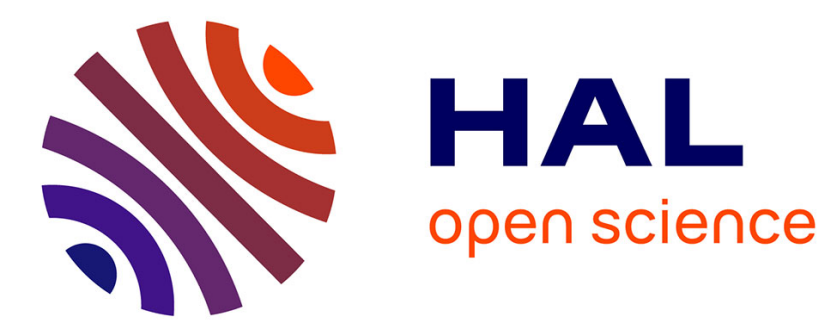

\title{
Picosecond Dynamics Behind the Shock Front
}

D. Dlott

\section{- To cite this version:}

D. Dlott. Picosecond Dynamics Behind the Shock Front. Journal de Physique IV Proceedings, 1995, 05 (C4), pp.C4-337-C4-343. 10.1051/jp4:1995426 . jpa-00253725

\section{HAL Id: jpa-00253725 https://hal.science/jpa-00253725}

Submitted on 1 Jan 1995

HAL is a multi-disciplinary open access archive for the deposit and dissemination of scientific research documents, whether they are published or not. The documents may come from teaching and research institutions in France or abroad, or from public or private research centers.
L'archive ouverte pluridisciplinaire HAL, est destinée au dépôt et à la diffusion de documents scientifiques de niveau recherche, publiés ou non, émanant des établissements d'enseignement et de recherche français ou étrangers, des laboratoires publics ou privés. 


\title{
Picosecond Dynamics Behind the Shock Front
}

\author{
D.D. Dlott
}

School of Chemical Sciences, University of Illinois at Urbana Champaign, Box 37-1 Noyes Lab, 505 S. Mathews Ave., Urbana, IL 61801, U.S.A.

\begin{abstract}
Understanding the microscopic details of shock-wave initiation of energetic materials requires a realistic picture of picosecond time scale processes occurring in large molecules located in an 100 nanometer thin layer just behind the front. In this paper, I discuss a theoretical model for shock wave induced chemistry which highlights the role of molecular mechanical energy transfer processes, especially multiphonon up-pumping. Picosecond laser measurements of multiphonon up-pumping in a high explosive, nitromethane, are presented. Up-pumping occurs on the $\sim 100$ picosecond time scale in nitromethane. The fundamental problem in direct measurement of the states of molecules just behind a shock front is simultaneously achieving picosecond time resolution and nanometer space resolution. A new method for probing dynamics immediately behind a shock front using optical nanogauges is described, and some preliminary results are presented.
\end{abstract}

\section{INTRODUCTION}

Little is known about the very first events occurring just behind the shock front during shock-induced initiation of condensed phase explosives. Because typical velocities for initiating shock waves are on the order of $3-6 \mathrm{~km} / \mathrm{s}$, and $1 \mathrm{~km} / \mathrm{s}=1 \mathrm{~nm} / \mathrm{ps}$, material within $100 \mathrm{ps}$ of the shock front is present in a very thin layer whose thickness is a few hundred nanometers. In this paper, I will discuss theoretical models and experimental measurements of condensed matter molecular dynamics in this layer. Both the theoretical and experimental investigations face daunting obstacles. To develop an adequate theory, one has to know much about the detailed microscopic dynamics of rather large and complicated molecules under harsh, extreme conditions which are evolving on the picosecond or even femtosecond time scales. To investigate these phenomena experimentally, one needs to use spectroscopic techniques with good time resolution, which is straightforward today, but one must also combine extremely high spatial resolution, ideally much better than a wavelength of visible light.

In this paper, I will concentrate on the problem of molecular mechanical energy transfer behind the shock front [1]. As shown in Fig. 1, in the long term a shock wave produces a jump in the temperature and pressure. But in the short term, the molecules just behind the shock front are far from mechanical and thermal equilibrium [1,2]. The motivation for studying picosecond dynamics behind a shock front is to learn more about the behavior of molecules far from equilibrium, to relate this behavior to the sensitivity of energetic materials to shock excitation, and to attempt to develop a detailed picture of the first few nanoseconds of the initiation process. 
The paper is divided into three parts. In the first part, I discuss the elements of a theoretical model developed in collaboration with Prof. M. D. Fayer and Dr. A. Tokmakoff of Stanford University. In the second part, I discuss experimental measurements of picosecond time scale energy transfer dynamics in a high explosive. The third part describes some new techniques which use optical shock nanogauges to probe the behavior of molecules in the nanometerthick layer just behind the shock front.

\section{THEORETICAL MODEL}

The details of this model have been described in two recent publications [1], so only the basic elements are given here. The model is intended to describe how the passage of a shock wave through an elastic solid causes chemical reactions to occur in energetic materials which, for widely used secondary explosives, are crystals consisting of relatively large molecules. The restriction to elastic solids means that hydrodynamic phenomena such as shear or moving edge dislocations are not considered. Although these phenomena may be quite important in many circumstances, the intent of the model is to understand chemistry caused by the weakest shock waves possible, because the critical issues in sensitivity involve weak shock waves characteristic of accidents. (a)

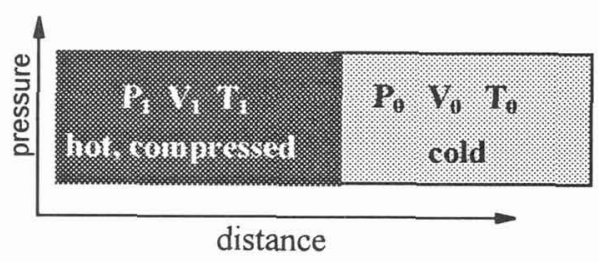

(b) reaction ignition up-pumping

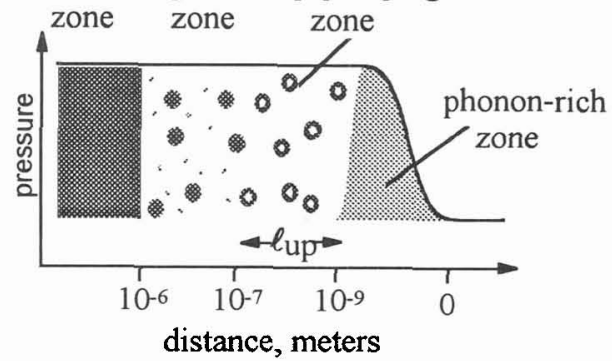

Figure 1. (a) A shock wave ultimately causes a jump in $T, P$. (b) Immediately behind the front, the material is not in thermal equilibrium. Reproduced from ref. 1.

The fundamental idea is that shock waves do not instantaneously produce Boltzmann equilibrium. Instead the shock front preferentially excites phonons (see Fig. 2). Subsequently these phonons excite intramolecular vibrations by the process termed multiphonon up-pumping $[1,3]$. The vibrational states must be excited before thermochemical reactions can occur [1]. Immediately behind the shock front is a thin layer which is phonon-rich and vibrationstarved. Arrhenius kinetics are not valid in this up-pumping zone (Fig. 1b). The up-pumping problem is han-

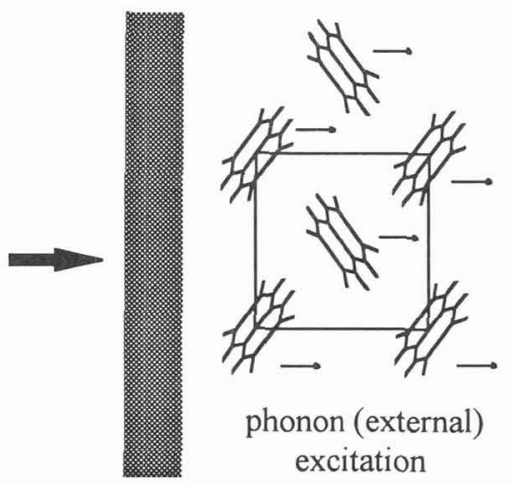

Figure 2. The shock first excites phonons, which are states which couple efficiently to volume perturbations. Molecular vibrations are excited later by multiphonon up-pumping.

dled using a simplification termed the quasitemperature model [1]. In this model, the phonon excitation level is characterized by a time-dependent phonon quasitemperature $\theta_{p}(t)$, and the vibrational excitation by a time-dependent vibrational quasitemperature $\theta_{\text {vib }}(t)$.

For a given thin layer of energetic material, we denote the zero of time to occur when the shock front reaches the layer. For a shock which ultimately heats the material from $T_{0}$ to $T_{1}$, the initial conditions are $\theta_{\text {vib }}(\mathrm{t})=\mathrm{T}_{0}$ and $\theta_{\mathrm{p}}(\mathrm{t}) \gg \mathrm{T}_{1}$. In other words, efficient shock-to-phonon transfer results in an 
instantaneous zero-time value of $\theta_{p}(t)$ which is much greater than $T_{1}$. This temporarily massive phonon overheating is a central feature of our model [1].

Phonons up-pump vibrations via anharmonic coupling [1,3]. In a large molecule, there are many different pathways for up-pumping, and in order to have a tractable model, we need to know which pathways dominate. Experimental studies of picosecond time scale vibrational energy transfer [4] show the principal mechanism of up-pumping involves the lowest-order or cubic anharmonic coupling. In the cubic coupling mechanism, the lowest energy vibrations, termed doorway states, are excited by simultaneously absorbing two phonons. Subsequently, molecules with excited doorway states can be pumped to higher vibrational levels in many steps, each involving absorption of a single phonon [1].

By using reasonable values for anharmonic coupling and density of states parameters, we have computed the rate of up-pumping, and the width of the up-pumping zone behind the shock front (Fig. 1b), as a function of the compression ratio, as shown in Fig. 3 [1]. We estimated the width of this zone to be $\sim 100 \mathrm{~nm}$, and the up-pumping time constant to be $30-100 \mathrm{ps}$ [1].

We have also investigated how energy transfer can affect the sensitivity of energetic materials. There is no a priori reason why up-pumping alone should directly affect sensitivity. With up-pumping there is simply a brief delay time of $\sim 100 \mathrm{ps}$ between the passage of the shock front and the onset of activation of the molecular vibrations. Nevertheless, recent calculations by Fried and Ruggerio [5] led them to suggest that the most insensitive energetic materials have the slowest up-pumping processes and the most sensitive materials have the fastest up-pumping. Although that work is still in its early stages, the initial result is certainly intriguing. Two quite different ways in which up-pumping can affect sensitivity have been suggested. L. Fried has pointed out that more efficient up-

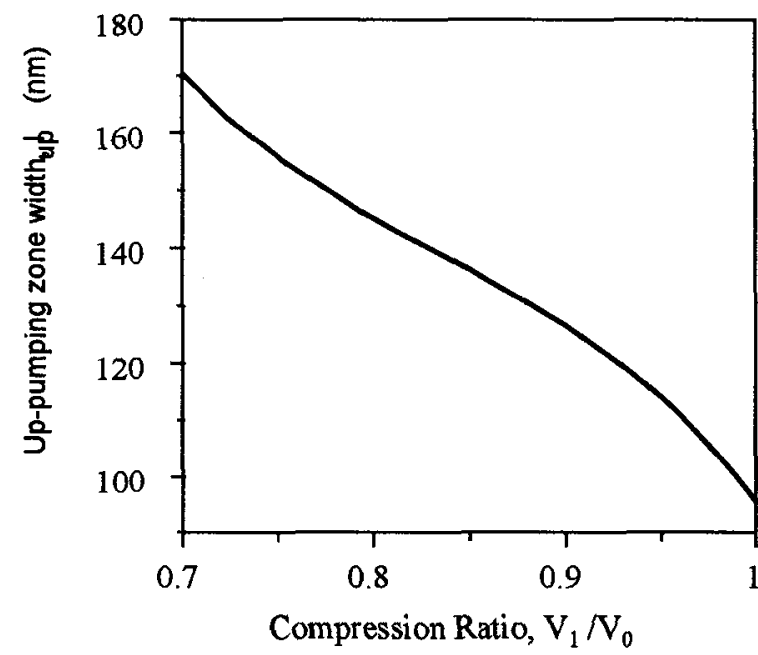

Figure 3. Computed width of the up-pumping zone. Reproduced from reference 1. pumping is a sign that the molecular vibrations are more efficiently coupled to the bath [6]. In Kramer's theory of condensed phase reaction dynamics, there exists a weak-coupling limit where increased coupling to the bath increases the rates of chemical reactions.

Dlott and Fayer proposed that the magnitudes of anharmonic couplings near defect-perturbed domains (DPD) in an imperfect solid might differ from couplings in the bulk [1]. The parameter $\xi$ is the magnitude of the anharmonic coupling enhancement. For DPD's where $\xi>1$, the local anharmonic coupling exceeds the bulk coupling. In these DPD's, localized temporary hot spots can be produced. Hot spots are produced because the phonons pump these DPD's faster than they pump the bulk vibrations. Because chemical reactions are highly sensitive to temperature, the reactivity of these hot spots can be much greater than the bulk reactivity. Using chemical reaction rate laws for typical energetic materials, we calculated Fig. 4. In Fig. 4, we assumed a $4.7 \mathrm{GPa}$ shock wave. The heating caused by this shock wave causes a bulk reaction probability of $\sim 10^{-10}$. This means one molecule in $10^{10}$ reacts, which is hardly enough to initiate widespread reactions. But just a small amount of anharmonic enhancement, e.g. $\xi=2$ in Fig. 4 can cause the defect reaction probability to increase by eight orders of magnitude [1]. 
When these theoretical models were proposed, we pointed out the need for experimental measurements of up-pumping time constants, and a better understanding of the role of DPD's in energy transfer, specifically the relationships between DPD structure and anharmonic coupling [1]. Such experiments are quite difficult and they require the development of new techniques, especially using time-resolved nonlinear optical phenomena.

\section{DIRECT OPTICAL MEASURE- MENT OF UP-PUMPING IN HIGH EXPLOSIVES}

Although many different techniques have been developed for studying vibrational relaxation and vibrational cooling in molecular systems, measurements of up-pumping are more problematic. In a vibrational relaxation measurement, a higher energy vibration is excited by a short laser pulse. The decay of energy (or coherence) of this state is then relatively easy to monitor because the laser-induced excitation is far greater than the equilibrium level of excitation [4]. In up-pumping studies, a method must be found for instantaneously producing a huge excess of phonons. The phonon excess must be sufficient to drive a substantial number of the molecule's vibrations to an excitation level which can be readily detected.

We have solved the phonon excitation problem by using a unique molecular dye [7] termed IR-165. This near-IR dye is useful because: (1) its maximum absorption is near $\lambda=1 \mu \mathrm{m}$, which is the wavelength of the most efficient solid state laser systems, Nd:YAG and Nd:YLF; (2) after absorbing a near-IR photon, efficient internal conversion and vibrational cooling processes result in a nearly total conversion of the optical energy into heat on the 4 ps time scale [8]; and (3) the mechanical energy lost by this dye excites mainly phonons in the surroundings $[7,8]$.

In our experiments [9], we dissolved a small amount ( 1\%) of IR-165 dye in nitromethane (NM). A $1 \mathrm{~mJ}, 1.053 \mu \mathrm{m}$ pulse of 75 ps duration pumps the dye, producing a burst of phonons. The pumping process deposits enough energy into a uniformly heated volume to ultimately produce a temperature jump of $\Delta \mathrm{T} \sim 25$ degrees. A visible, $0.527 \mu \mathrm{m}$ pulse then probes the excited volume. This pulse produces incoherent anti-Stokes Raman emission, which is collected and analyzed with a spectrometer and a photodetector. The anti-Stokes emission from a given vibration is proportional to the instantaneous occupation number of the state. Thus anti-Stokes probing is mode specific, and it allows us to quantitatively measure the change in occupation number $\Delta \mathrm{n}_{\omega}(\mathrm{t})$ for a particular NM vibration. For convenience, we mathematically converted this occupation number into a time-dependent vibrational quasitemperature increase $\Delta \theta_{\omega}(\mathrm{t})$, using the relation, $\Delta \mathrm{n}_{\omega}(\mathrm{t})=\left[\exp \left(\hbar \omega / \mathrm{k} \Delta \theta_{\omega}\right)-1\right)^{-1}$. Figure 5 shows our results on NM [9].

In our experiments we probed two states of $\mathrm{NM}$, the $657 \mathrm{~cm}^{-1}$ vibration, which is assigned to a symmetric bending vibration of the $\mathrm{NO}_{2}$ group, and the $918 \mathrm{~cm}^{-1}$ vibration, assigned to a $\mathrm{C}-\mathrm{N}$ stretching motion [9]. The latter vibration is of much interest, since many theoretical models of NM ignition focus on the process of phonon-activation of the $\mathrm{C}-\mathrm{N}$ stretching vibration, followed by cleavage of the $\mathrm{C}-\mathrm{N}$ bond. 
There are two interesting features in Fig. 5. The duration of the up-pumping period is about $100 \mathrm{ps}$, and vibrations are excited sequentially; we observed an $\sim 30$ ps delay between the excitation of the lower energy vibration and the excitation of the higher energy vibration. This experiment [9] provides the first direct measurement of multiphonon uppumping in high explosives. The results are generally consistent with the theoretical model described in the previous section, namely that uppumping occurs on the $\sim 100 \mathrm{ps}$ time scale, and it is the result of phonons first pumping doorway modes, and then later pumping higher energy vibrations.

\section{PICOSECOND DYNAMICS BE- HIND THE SHOCK FRONT}

There are many technical obstacles to studying picosecond energy transfer dynamics behind a shock front. The principle problems are: (1) generating reproducible solid-state shock waves at a reasonable repetition rate; (2) probing the shocked material with a method which provides detailed information about energy transfer and chemical reactivity, and (3) localizing the probe measurement to a nanometer-scale region behind the front, and accurately synchronizing the measurement to the arrival of the shock front at this layer.

There are many options to deal with problems (1) and (2), but until just recently problem (3) seemed insoluble. The principle obstacle is that time-resolved optical measurements are limited in principle by diffraction to regions of micron thickness, and in practice, depth-of-field considerations and edge effects give even worse spatial resolution. Our novel approach to this problem involves the use of shock target arrays with optical nanogauges. The nanogauge system is shown in Fig. 6.

In our experiments, a picosecond near-IR pulse produces shock waves at a repetition rate of 10 shocks/s, using an exploding confined foil generator developed by workers at the Naval Research Laboratory [10]. The pulse travels

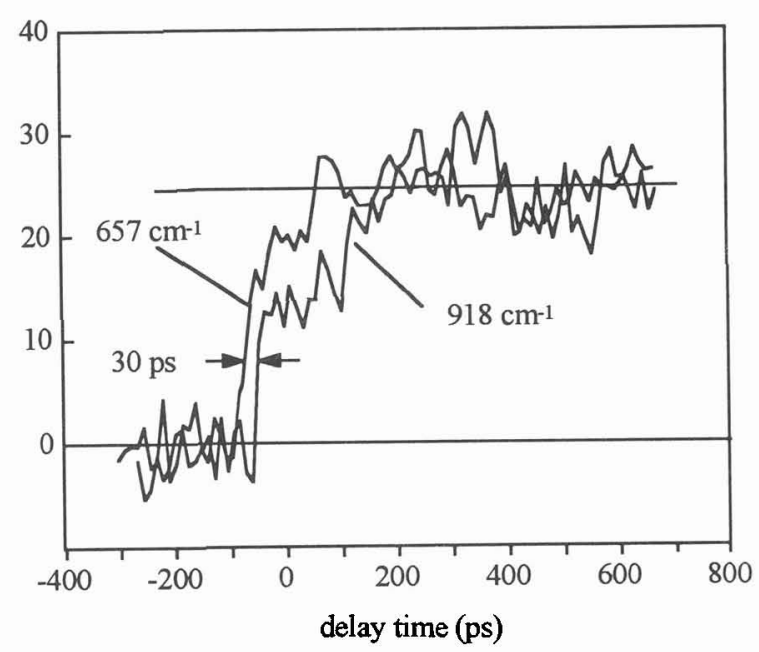

Figure 5. Multiphonon up-pumping in nitromethane (NM). Uppumping occurs on the $100 \mathrm{ps}$ time scale. Higher energy vibrations become excited later. The $y$-axis is the increase in quasitemperature of each vibration.

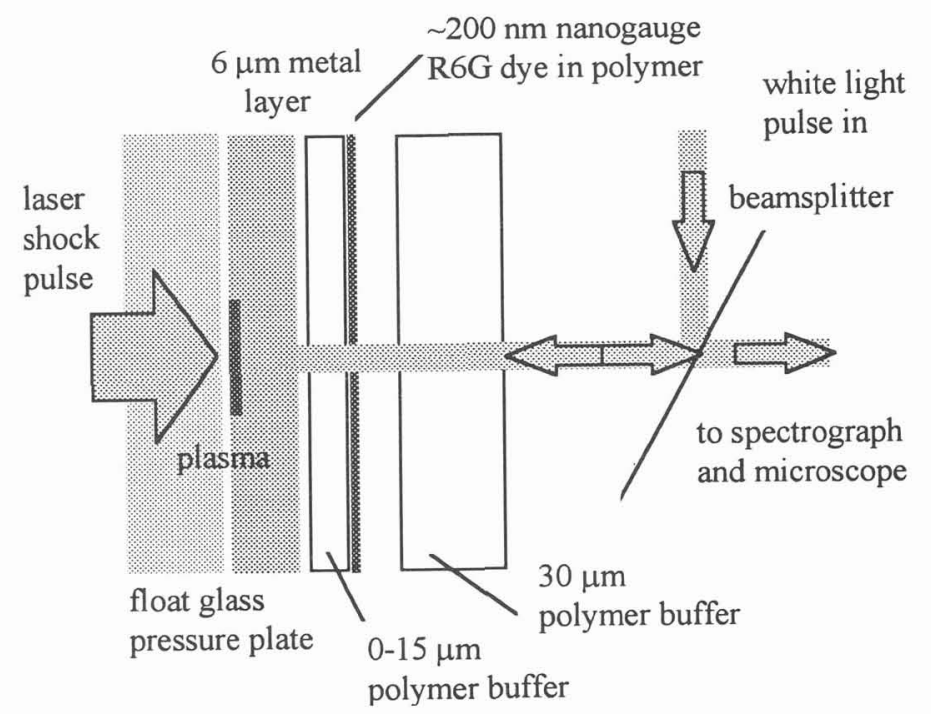

Figure 6. Schematic drawing (not to scale) of one element of a shock target array. Rapid expansion of the laser-driven plasma drives a shock wave into the polymer layers. A picosecond whitelight pulse observes the instantaneous absorption spectrum of the optical nanogauge, consisting of a dye in a polymer thin film. 
through a glass pressure plate, and it is weakly absorbed by a thin metal layer. When the surface of this layer is hot enough to form a strongly absorbing plasma, the remainder of the pulse intensely heats the plasma. Rapid expansion of the plasma drives a shock wave through the metal layer into the sample region [10], which is a multilayer polymer thin film [11]. After traveling a few $\mu \mathrm{m}$ through polymer, the shock front hits the $\sim 200 \mathrm{~nm}$ thick optical nanogauge. In our initial experiments [11], the nanogauge consisted of an absorbing dye, R6G. Energy transfer in R6G, occurring as a result of the shock excitation, is monitored using a ps-duration white light pulse. This white probe pulse does a double-pass through the nanogauge, reflecting once off the metal layer. The probe is then sent to an optical microscope, or to a multichannel spectrograph. The large-area array target has about $10^{5}$ individually adressible shock pixels. A motorized positioner is used to translate the sample through the axis of the laser pulses, so a fresh target region is used for every shot. The details of this technique are reported in ref. 11.

With the microscopy apparatus, we can detect the arrival of the shock wave at the metal-polymer interface, and the arrival of the shock at the optical nanogauge. By knowing the thickness of the polymer buffer layer in Fig. 6, we accurately determined the shock velocity, which was $5.5 \mathrm{~km} / \mathrm{s}$, giving a shock pressure of $\sim 15 \mathrm{GPa}$ [11]. Because the white probe pulse is seeing only effects attributed to the absorption of the $\mathrm{R} 6 \mathrm{G}$ dye, we have, by microfabrication of this array, limited the region of observation to a nanometer thick volume.

Figure 7 shows an unshifted, and shock-shifted spectrum of R6G, taken with our apparatus [12]. Shock-induced spectroscopy of dye molecules is not new [13], but there is a critical difference in our

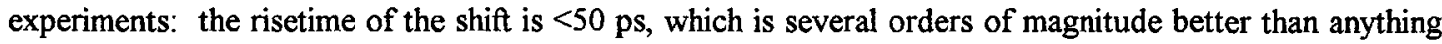
observed previously. Besides the shift, there are subtle changes in the red-edge of the optical absorption which can be used to investigate the redistribution of vibrational energy in the dye molecules during shockinduced up-pumping [14].

\section{CONCLUDING REMARKS}

Now that we have found experimental solutions to the fundamental problems of studying picosecond time scale energy transfer behind a shock front, we intend to extend these methods to study energetic materials under conditions typical of shock initiation processes. Most interesting energetic materials do not have the intense visible absorption of $\mathrm{R} 6 \mathrm{G}$, so a different probing technique is needed. We have recently been able to show that picosecond time-resolved coherent Raman scattering (ps CARS) can be used to study the vibrational dynamics and chemical reactions of colorless materials just behind a shock front [15], We have considerable optimism that direct ultrafast time scale measurements of energetic materials behind a

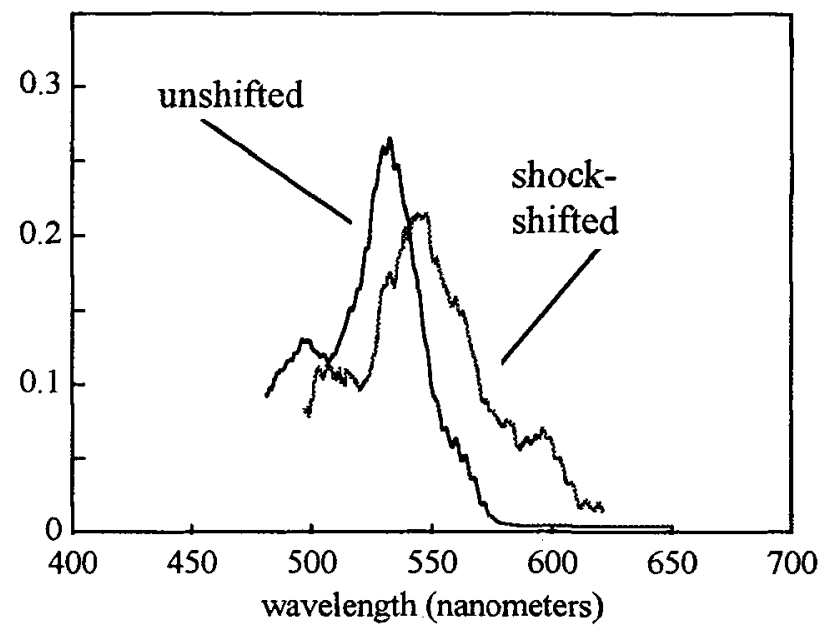

Figure 7. Spectrum of R6G optical nanogauge, taken using the apparatus in Fig. 6. The unshifted spectrum was taken a few tens of ps before the shock front reached the nanogauge. The red-shifted spectrum was taken about 100 ps after the front reached the nanogauge. The red shift is consistent with the $\sim 15 \mathrm{GPa}$ pressure of the shock wave. 
shock front will be accomplished in the near future. These measurements promise to open a new window onto the picosecond dynamics behind the shock front, which we believe will be key in understanding the initiation of energetic materials at the molecular level.

\section{ACKNOWLEDGMENT}

This research was supported by US Air Force Office of Scientific Research grant F49620-94-0108, US Army Research Office grant DAAH04-93-G-0016, and National Science Foundation grant DMR-9404806. The work would not have been possible without the efforts of many valued collaborators. The shockinduced chemistry model was developed with Prof. Michael D. Fayer and Dr. Andrei Tokmakoff of Stanford University. The nitromethane measurements were performed by Dr. Sheah Chen and Dr. Xiaoyu Hong at Illinois and Dr. William Tolbert, presently at $3 \mathrm{M}$ Corporation. The shock measurements were made by Dr. I-Yin Sandy Lee and Dr. Jeffrey R. Hill at Illinois.

\section{REFERENCES}

[1] D. D. Dlott and M. D. Fayer, J. Chem. Phys. 92 (1990) 3798; A. Tokmakoff, M. D. Fayer and D. D. Dlott, J. Phys. Chem. 97 (1993) 1901.

[2] C. S. Coffey and E. T. Toton, J. Chem. Phys. 76 (1982) 949; F. J. Zerilli and E. T. Toton, Phys. Rev. B 29 (1984) 5891; R. D. Bardo, Int. J. Quantum Chem. 20 (1986) 455.

[3] H. Kim and D. D. Dlott, J. Chem. Phys. 93 (1990) 1695.

[4] D. D. Dlott, in Laser Spectroscopy of Solids II; Topics in Applied Physics, vol. 65, W. Yen, ed. (Springer Verlag: Berlin; 1989), Chapter 5.

[5] L. Fried and A. Ruggerio, J. Phys. Chem. 98 (1994) 9786.

[6] L. Fried, personal communication.

[7] S. Chen, I-Y. S. Lee, W. A. Tolbert, X. Wen and D. D. Dlott, J. Phys. Chem. 96 (1992) 7178.

[8] X. Wen, W. A. Tolbert and D. D. Dlott, J. Chem. Phys. 99 (1993) 4140.

[9] S. Chen, W. A. Tolbert and D. D. Dlott, J. Phys. Chem. 98 (1994) 7759.

[10] A. L. Huston, B. L. Justus and A. J. Campillo, Chem. Phys. Lett. 118 (1985) 267; B. L. Justus, A. L. Huston and A. J. Campillo, ibid, 128 (1986) 274; B. L. Justus, C. D. Merritt and A. J. Campillo, ibid, 156 (1989) 64; B. L. Justus, A. L. Huston, and A. J. Campillo, Appl. Phys. Lett. 47 (1986) 1159.

[11] I-Y. S. Lee, J. R. Hill, and D. D. Dlott, J. Appl. Phys. 75, (1994) 2975.

[12] I-Y. S. Lee, J. R. Hill, and D. D. Dlott, (unpublished work).

[13] X. A. Shen and Y. M. Gupta, J. Appl. Phys. 70 (1991) 7549.

[14] X. Wen, W. A. Tolbert and D. D. Dlott, Chem. Phys. Lett. 192 (1992) 315.

[15] D. E. Hare and D. D. Dlott, Appl. Phys. Lett. 64 (1994) 715; D. E. Hare, J. Franken, D. D. Dlott, E. L. Chronister and J. J. Flores, Appl. Phys. Lett. (in press, 1994). 